\title{
QTLs for the elongation of axile and lateral roots of maize in response to low water potential
}

\section{Journal Article}

Author(s):

Ruta, N.; Liedgens, M.; Fracheboud, Y.; Stamp, Peter; Hund, Andreas (1)

Publication date:

2010

Permanent link:

https://doi.org/10.3929/ethz-b-000017297

Rights / license:

In Copyright - Non-Commercial Use Permitted

Originally published in:

Theoretical and Applied Genetics 120(3), https://doi.org/10.1007/s00122-009-1180-5 


\title{
QTLs for the elongation of axile and lateral roots of maize in response to low water potential
}

\author{
N. Ruta $\cdot$ M. Liedgens $\cdot$ Y. Fracheboud $\cdot$ \\ Peter Stamp $\cdot$ A. Hund
}

Received: 13 January 2009/Accepted: 4 October 2009/Published online: 22 October 2009

(C) Springer-Verlag 2009

\begin{abstract}
Changes in root architecture and the maintenance of root growth in drying soil are key traits for the adaptation of maize (Zea mays L.) to drought environments. The goal of this study was to map quantitative trait loci (QTLs) for root growth and its response to dehydration in a population of 208 recombinant inbred lines from the International Maize and Wheat Improvement Center (CIMMYT). The parents, Ac7643 and Ac7729/TZSRW, are known to be drought-tolerant and drought-sensitive, respectively. Roots were grown in pouches under wellwatered conditions or at low water potential induced by the osmolyte polyethylene glycol (PEG 8000). Axile root length $\left(L_{\mathrm{Ax}}\right)$ increased linearly, while lateral root length $\left(L_{\text {Lat }}\right)$ increased exponentially over time. Thirteen QTLs were identified for six seedling traits: elongation rates of axile roots $\left(\mathrm{ER}_{\mathrm{Ax}}\right)$, the rate constant of lateral root elongation $\left(k_{\mathrm{Lat}}\right)$, the final respective lengths $\left(L_{\mathrm{Ax}}\right.$ and $\left.L_{\mathrm{Lat}}\right)$, and the ratios $k_{\mathrm{Lat}} / \mathrm{ER}_{\mathrm{Ax}}$ and $L_{\mathrm{Lat}} / L_{\mathrm{Ax}}$. While QTLs for lateral root traits were constitutively expressed, most QTLs for axile root traits responded to water stress. For axile roots, common QTLs existed for $\mathrm{ER}_{\mathrm{Ax}}$ and $L_{\mathrm{Ax}}$. Quantitative trait loci for the elongation rates of axile roots responded more clearly to water stress compared to root length. Two major QTLs were detected: a QTL for general vigor in bin 2.02,
\end{abstract}

Communicated by B. Godshalk.

N. Ruta $\cdot$ M. Liedgens $\cdot$ P. Stamp $\cdot$ A. Hund $(\bowtie)$

Institute of Plant Science, ETH Zurich, 8092 Zurich, Switzerland

e-mail: andreas.hund@ipw.agrl.ethz.ch

P. Stamp

e-mail: peter.stamp@ipw.agrl.ethz.ch

Y. Fracheboud

Umea Plant Science Center, Department of Plant Physiology,

University of Umeå, 90187 Umeå, Sweden affecting most of the traits, and a QTL for the constitutive increase in $k_{\mathrm{Lat}}$ and $k_{\mathrm{Lat}} / \mathrm{ER}_{\mathrm{Ax}}$ in bins 6.04-6.05. The latter co-located with a major QTL for the anthesis-silking interval (ASI) reported in published field experiments, suggesting an involvement of root morphology in drought tolerance. Rapid seedling tests are feasible for elucidating the genetic response of root growth to low water potential. Some loci may even have pleiotropic effects on yieldrelated traits under drought stress.

Keywords Drought - QTL · Root growth - Water potential · Drought resistance · Zea mays L. · Corn

\section{Introduction}

To deal with low water potential, plants have developed tolerance and avoidance mechanisms, which depend on the timing and severity of the stress (for terminology, see Verslues et al. 2006). During most drought events, crop plants avoid low water potential by achieving a balance between water uptake and water loss, e.g. by decreasing the stomatal aperture or by decreasing leaf growth rate while maintaining root growth. If plants cannot maintain this balance, they employ mechanisms to tolerate low water potential. These involve mechanisms to avoid dehydration, like the accumulation of solutes and osmotic adjustment.

The avoidance of low water potential by developing a greater rooting depth can explain an increase in grain yield of wheat (Triticum aestivum; Kirkegaard et al. 2007). Avoidance may be responsible for the historic yield increase in maize (Hammer et al. 2009) and adaptation of maize to drought environments (see Hund et al. 2009a). Several traits, which lead to a greater rooting depth, are under debate: a vertical orientation of the roots (Hammer 
et al. 2009) and a redirection of carbohydrates from lateral to axile roots (Hund et al. 2009a). A change in root morphology of maize because of adaptation to drought is supported by selection experiments. For example, root systems with a weaker development of crown (adventitious) and lateral roots (Bruce et al. 2002; Giuliani et al. 2005), a smaller amount of roots in the top $50 \mathrm{~cm}$ of the soil profile (Bolaños et al. 1993), as well as reduced extraction of water from the topsoil (Campos et al. 2004) show better adaptation to drought conditions.

Apart from a greater rooting depth per se, the maintenance of growth in drying soil (dehydration avoidance) may enable roots to penetrate deeper soil layers and, thus, enhances the avoidance of low water potentials by tapping new water supplies as suggested by the results of Sharp and Davies (1985). Compared to shoot growth, root growth in drying soil is less inhibited and, under mild stress, is even promoted (Sharp and Davies 1989). This suggests a different genetic control of the responses of roots and shoots optimized to enhance avoidance of low water potential.

While the traits of the root system rank high on the list of traits for improving the drought tolerance of maize (Campos et al. 2004; Ribaut et al. 2008), there is a lack of efficient screening systems to assess them. Comparing the root growth of genotypes in drying soil is extremely labor intensive and has the major disadvantage that differences in the water uptake among plants must be taken into account when comparing roots exposed to similar water potential. As an alternative, the effect of low water potential on root elongation can be studied by using osmolytes, such as polyethylene glycol (PEG) (Lagerwer et al. 1961). Polyethylene glycol with a molecular weight above 6,000 Da cannot penetrate the cell membranes of most species (Carpita et al. 1979); it is probably the best solute to reflect the type of stress imposed by drying soil (Verslues et al. 2006).

Architectural properties of the root system similarly expressed at early and at later developmental stages may be suitable targets for selection in crop improvement programs as pointed out by Manschadi et al. (2006, 2008). Moreover, the identification of QTLs controlling such traits enhances our understanding of their genetic control and their relationship with other important traits. The QTLs for root traits of maize seedling were mapped for their response to phosphorus (Zhu et al. 2005) and nitrate (Liu et al. 2008), under cool conditions (Hund et al. 2004), under hydroponic conditions (Tuberosa et al. 2002), and in growth pouches (Trachsel et al. 2009). Some of these studies attempted to link QTLs for root traits to QTLs for yield (Liu et al. 2008; Trachsel et al. 2009; Tuberosa et al. 2002). Here, we attempt to relate root growth to the above ground growth of plant organs.
The population described herein was previously used to map QTLs for the anthesis-silking interval (ASI) and yield components (Ribaut et al. 1996, 2007) as well as for the response of leaf elongation to water deficit (Welcker et al. 2007). A possible explanation of a drought-induced increase in the ASI is a reduction in the elongation rate of the silks (Fuad-Hassan et al. 2008). Indeed, the results of Welcker et al. (2007) suggest that the growth of silks and leaves have common genetic determinisms. Roots may be linked to the maintenance of leaf and silk growth in three ways: (1) by a change in root architecture enabling roots to explore a large soil volume, while minimizing the resistance to water flux through the soil (Manschadi et al. 2006; Tardieu et al. 1992) to avoid low root water potential, (2) by maintaining root growth at low water potential enabling roots to explore new water sources, and (3) by strategies to avoid dehydration, thus enabling the growth of plant organs.

As outlined above, we identified the relationship between axile and lateral roots as a candidate trait for improving the drought tolerance of maize (Hund et al. 2009a) and developed a method for the rapid, nondestructive assessment of the growth of both root types in growth pouches (Hund et al. 2009 b). The objectives were (1) to study the dynamics of elongation of axile and lateral roots and their response to low water potential and to identify QTLs related to these traits; (2) to compare the modeled root growth with root length at the end of the study period; (3) to compare QTLs for these root traits with QTLs for ASI (Ribaut et al. 1996, 1997) and leaf elongation rates (Welcker et al. 2007).

\section{Materials and methods}

\section{Plant material}

From the cross between Ac7643 (P1) and Ac7729/TZSRW (P2), 208 RILs as well as the parental inbred lines were provided by CIMMYT. According to observations in the field, P1 is classified as having a short ASI and a relatively high yield under drought. By contrast, P2 is classified as having a long ASI and a relatively low yield under drought (Ribaut et al. 1996). Based on these results, we refer to P1 and P2 as being drought tolerant and drought sensitive, respectively.

\section{Growth conditions}

Seeds were germinated in the dark at $27^{\circ} \mathrm{C}$; healthy seedlings with a primary root about $1 \mathrm{~cm}$ long were transferred to growth pouches. These consisted of a blue germination blotter, $24 \times 29.5 \mathrm{~cm}$ (Anchor Paper, St. Paul, MI, USA), as the substrate for the growing roots and a black PE sheet (Walser AG, TG, Switzerland) as cover (see Hund et al. 
$2009 \mathrm{~b}$ for details). Growth pouches were hung in growth containers $(27 \times 37 \times 32 \mathrm{~cm})$. The containers were placed in a growth chamber (PGW36 Conviron, Winnipeg, MB, Canada) at $25^{\circ} \mathrm{C} / 22^{\circ} \mathrm{C}$ (day/night), $70 \% / 60 \%$ relative humidity (day/night), and a 12-h photoperiod with a photosynthetic active radiation of $400 \mu \mathrm{mol} \mathrm{cm} \mathrm{cm}^{-2} \mathrm{~s}^{-1}$. During the first 3 days after germination (DAG), all plants were grown with the lower edge of the pouch (about $2 \mathrm{~cm}$ high) submerged in a solution containing $0.23 \%$ (v/v) Wuxal (Aglukon Spezialdünger GmbH, Düsseldorf, Germany). Wuxal contains per liter $100 \mathrm{~g} \mathrm{~N}, 43 \mathrm{~g} \mathrm{P}, 62.5 \mathrm{~g} \mathrm{~K}, 190 \mathrm{mg}$ $\mathrm{Fe}, 162 \mathrm{mg} \mathrm{Mn}, 102 \mathrm{mg} \mathrm{B}, 81 \mathrm{mg} \mathrm{Cu}, 61 \mathrm{mg} \mathrm{Zn}$, and $10 \mathrm{mg}$ Mo. The growth containers were covered with aluminum laminated polystyrene (Spaarpor Klaus Eckhardt, GmbH, Neunkirchen, Germany) to protect the growth pouches from heating. After 3 days, all the pouches were submerged daily for $5 \mathrm{~min}$ in the basic medium solution (well watered, WW) or in the basic medium solution containing 20\% (w/v) PEG 8000 (Sigma-Aldrich $\mathrm{GmbH}$, Steinheim, Germany), thereafter referred to as water-stressed (WS). The predawn leaf water potential at harvest was measured with a plant water status console 3000 (Soil Moisture Equipment Corporation, Santa Barbara, CA, USA). The measurements were taken in the dark after the $12 \mathrm{~h}$ night on a set of nine randomly chosen genotypes per experimental run and treatment (one from each growth container; see below). The whole plant was cut at the shoot base and the shoot put into the cylinder of the plant water console. The pressure was increased slowly until the xylem water appeared at the cut section. The average predawn leaf water potential was $-0.09 \mathrm{MPa}(\mathrm{WW})$ and -0.74 MPa (WS) and the plants needed 7 (WW) and 9 days (WS) until their first leaf had fully developed (V1 stage).

\section{Root measurements}

The growth pouches in the WW and the WS treatments were scanned three $(3,5$, and $7 \mathrm{DAG})$ and four times $(3,5$, 7 , and 9 DAG), respectively. The images were preprocessed in Photoshop 7.0 (Adobe Systems Inc., San Jose, CA, USA) followed by digital image analysis in WinRHIZO (Regent Instruments, Quebec, Canada). Processing in Photoshop involved three steps. First, the saturation channel plugin Curvemeister 2 (Curvemeister, Berkeley, CA, USA) was used to generate 8-bit images, second, the median filter was used to remove background noise, and, third, an appropriate threshold was applied to separate roots from the background. The binary images were calculated in WinRHIZO (Regent Instruments, Montreal, QC, Canada). The debris removal filter was used to remove objects with an area smaller than $0.02 \mathrm{~cm}^{2}$ and a length/width ratio below 5. The diameter classes were set at $42 \mu \mathrm{m}$, the equivalent of one pixel. The root length in diameter-class distribution (RLDD) enabled us to distinguish the diameter classes belonging to lateral and axile roots. A diameter threshold of $0.546 \mathrm{~mm}$ was chosen to separate these two root types. The sum of the root length equal or below the threshold separating both root types was defined as lateral root length $\left(L_{\mathrm{Lat}}\right)$; the sum of the root length above the threshold was defined as axile root length $\left(L_{\mathrm{Ax}}\right)$. The ratio between lateral and axile roots $\left(L_{\mathrm{Lat}} / L_{\mathrm{Ax}}\right)$ was calculated from these measurements.

To determine whether the $L_{\mathrm{Lat}}$ and $L_{\mathrm{Ax}}$ elongated exponentially or more linearly over time, the samples of the two parental lines were analyzed with the function gls from the $\mathrm{R}$ package nlme (Pinheiro et al. 2007). This package enables us to account for the non-homogenous variance and autocorrelation of the residuals. The initial formulation of the statistical model for both root types was as follows:

$$
\begin{aligned}
y_{i j k}= & p_{i}+w_{j}+t_{k}+t_{k}^{2}+p_{i} w_{j}+p_{i} t_{k}+w_{j} t_{k}+p_{i} w_{j} t_{k} \\
& +p_{i} t_{k}^{2}+w_{j} t_{k}^{2}+p_{i} w_{j} t_{k}^{2}+e_{i j k},
\end{aligned}
$$

where $y_{i j k}$ is the measured root length of the parental line $p_{i}$, $w_{j}$ the water treatment, sample at time $t_{k}$ and $e_{i j k}$ is the residual error. The final formulation of the model resulted from backward selection based on the $P$ value with a probability threshold at 0.05 . During backward selection, marginality was accounted for, i.e. non-significant main effects were retained in the model if any of the interaction terms, including the target main effect, were significant.

According to the results of model 1, the elongation rate of the axile roots $\left(\mathrm{ER}_{\mathrm{Ax}}\right)$ and the rate constant of lateral root elongation $\left(k_{\mathrm{Lat}}\right)$ were determined. Both are referred to as elongation rates to simplify the discussion. The corresponding model for the axile roots was:

$x(t)=x\left(t_{0}\right)+\mathrm{ER}_{\mathrm{Ax}} t ; \quad \mathrm{ER}_{\mathrm{Ax}}=\frac{x(t)-x\left(t_{0}\right)}{t}$,

where $x(t)$ is the root length at time $t$ after germination and $x\left(t_{0}\right)$ is the root length on the first day of scanning (DAG 3). The model for the lateral roots was:

$x(t)=x\left(t_{0}\right) \times e^{k_{\text {lat }} t} ; \quad k_{\text {lat }}=\frac{\log (x(t))-\log \left(x\left(t_{0}\right)\right)}{t}$

The rate constant $k_{\mathrm{Lat}}$ is inversely proportional to the doubling time of the lateral roots. The ratio between $k_{\text {Lat }}$ and $\mathrm{ER}_{\mathrm{Ax}}$ was calculated $\left(k_{\mathrm{Lat}} / \mathrm{ER}_{\mathrm{Ax}}\right)$. Leaf area was measured with a LI-3000A area meter (LICOR, Inc., Lincoln, NE, USA).

Experimental design and statistics

The experimental design was an alpha lattice $(0,1)$ design (Barreto et al. 1997) with six independent runs, i.e. replications (Rep), 216 treatment factors (208 RILs and $4 \times 2$ parents), and 24 plots per incomplete block, consisting of a 
pair of growth containers, one for WW and one for WS, respectively. Each of the paired growth containers contained the same set of 12 genotypes. Nine pairs of growth containers were placed in each of two growth chambers. In this design, 12 plants were measured for each genotype, 6 plants for each water treatment. Treatment and replication effects were considered to be fixed, while incomplete blocks nested within growth chambers and replications were considered to be random. Analysis of variance was done using the R package ASREML (Butler et al. 2007) and the best linear unbiased predictors (BLUPs), extracted for each genotype $\mathrm{x}$ treatment combination, were used as the input values for the QTL mapping. Outliers were identified according to Chauvenet's criterion, i.e. observations with a standardized residual greater than 3.7 (the exact value depended on the number of observations) were discarded from the analysis. The broad-sense heritability for each treatment was calculated according to Hallauer and Miranda (1981) as

$H^{2}=\frac{\sigma_{g}^{2}}{\sigma_{g}^{2}+\frac{1}{b} \sigma_{e}^{2}}$,

where $\sigma_{g}^{2}$ is the genetic variance, $\sigma_{e}^{2}$ is the residual error variance, and $b$ is the number of replications.

\section{QTL analysis}

The QTLs were identified using the RFLP linkage map published by Fracheboud et al. (2002). The map consisted of 132 RFLP markers with a total distance of 2,250 cM and an average distance of $17.1 \mathrm{cM}$. The QTLs were detected by composite interval mapping using QTL Cartographer 1.17 model 6 (Basten et al. 2003), with a blocking window size of $30 \mathrm{cM}$. The co-factors were selected by forward and backward regressions with in and out thresholds at a $P$ value of 0.01 . Data of each trait from both WW and WS treatments were analyzed jointly in a combined analysis (Jiang and Zeng 1995), allowing for the determination of the QTL-by-environment interaction (QEI). A QTL was considered to be significant when the joint LOD score was higher than 3. The detected QTLs were considered to be significant in the individual experiments when the corresponding LOD score was higher than 2.5. The thresholds represent a comparison-wise alpha significance value of 0.06 and an experiment-wise alpha significance value of 0.003 , assuming that all 20 chromosome arms segregate independently. The corresponding LOD score for QEI was 0.8. The support interval of a QTL was defined as the segment of the chromosome, in which the LOD at the peak decreased by half. Multiple regressions were used to evaluate the total percentage of phenotypic variation accounted for by all the identified QTLs.

\section{Results}

Principle growth dynamics of axile and lateral root length

In order to achieve a normal distribution of the residuals for lateral root length, but not for axile root length, a logarithmic transformation was required. For both types of root length, it was necessary to account for non-constant variance in the residuals. It was modeled with an exponential function either of the fitted values or of time for the axial root length and the lateral root length, respectively (data not shown). Accounting for auto-correlation, which was expected because of the repeated measurement of the same samples, did not improve the model, so the more parsimonious formulation, without estimation of the correlation among repeated measurements, was retained.

Axial root length increased linearly (Fig. 1a) during the experiment, and neither a logarithmic transformation of the length data, nor a quadratic term (Table 1), was required for modeling the data (Eq. 2). Lateral root length increased exponentially (Fig. 1b), which was accounted for by the logarithmic transformation of the data (Eq. 3). The corresponding Tukey-Anscombe plots (Fig. 1c, d) support the suitability of the models: the residuals are uniformly distributed around zero over the entire range of fitted values. This is made especially evident by the superimposed LOESS-fit, which closely follows the zero line for both fits. The highly significant quadratic term $(P=0.0015)$, resulting in a concave profile of the logarithm of the root length with time (data not shown), indicates that exponential growth rate is not constant over the course of this investigation.

For both root types, there was neither a significant effect of the water treatment nor an interaction with the parental lines. For lateral root length, there was also no effect of the genotype. Contrastingly, there was considerable $(P$ value $=0.0121)$ evidence that the length of the axial roots of $\mathrm{P} 2$ increase faster than that of P1.

Comparison of growth rates with root lengths at the end of the experiment

The final leaf area of the WS plants $\left(13 \mathrm{~cm}^{2}\right)$ was reduced by $38 \%$ compared to that of the WW plants $\left(21 \mathrm{~cm}^{2}\right.$ ) (data not shown). The slower growth in the WS treatment was much more pronounced for the shoots than for the roots. Under WS, the $\mathrm{ER}_{\mathrm{Ax}}$ decreased by $35 \%$ compared to WW, but the final $L_{\mathrm{Ax}}$ remained unchanged (Table 2). Somewhat similar effects were also found for lateral roots: $k_{\text {Lat }}$ decreased by $22 \%$ under WS, while $L_{\text {Lat }}$ was increased strongly (47\%). At the same developmental stage (V1), water-stressed plants were 2 days older than the well- 
Fig. 1 Change of axile roots length over time of Ac7643 (P1; solid lines) and Ac7729/ TZSRW (P2; dashed lines) (a); change of lateral root length over time for both parents (b). Lines represent the final models (Table 1). Tukey-Anscombe plots for axile root length of P1 (solid circles) and P2 (open circles) (c) and lateral roots length of both parents (d). Nonparametric LOESS-fit are superimposed (dashed lines; $\mathbf{c}, \mathbf{d}$ )
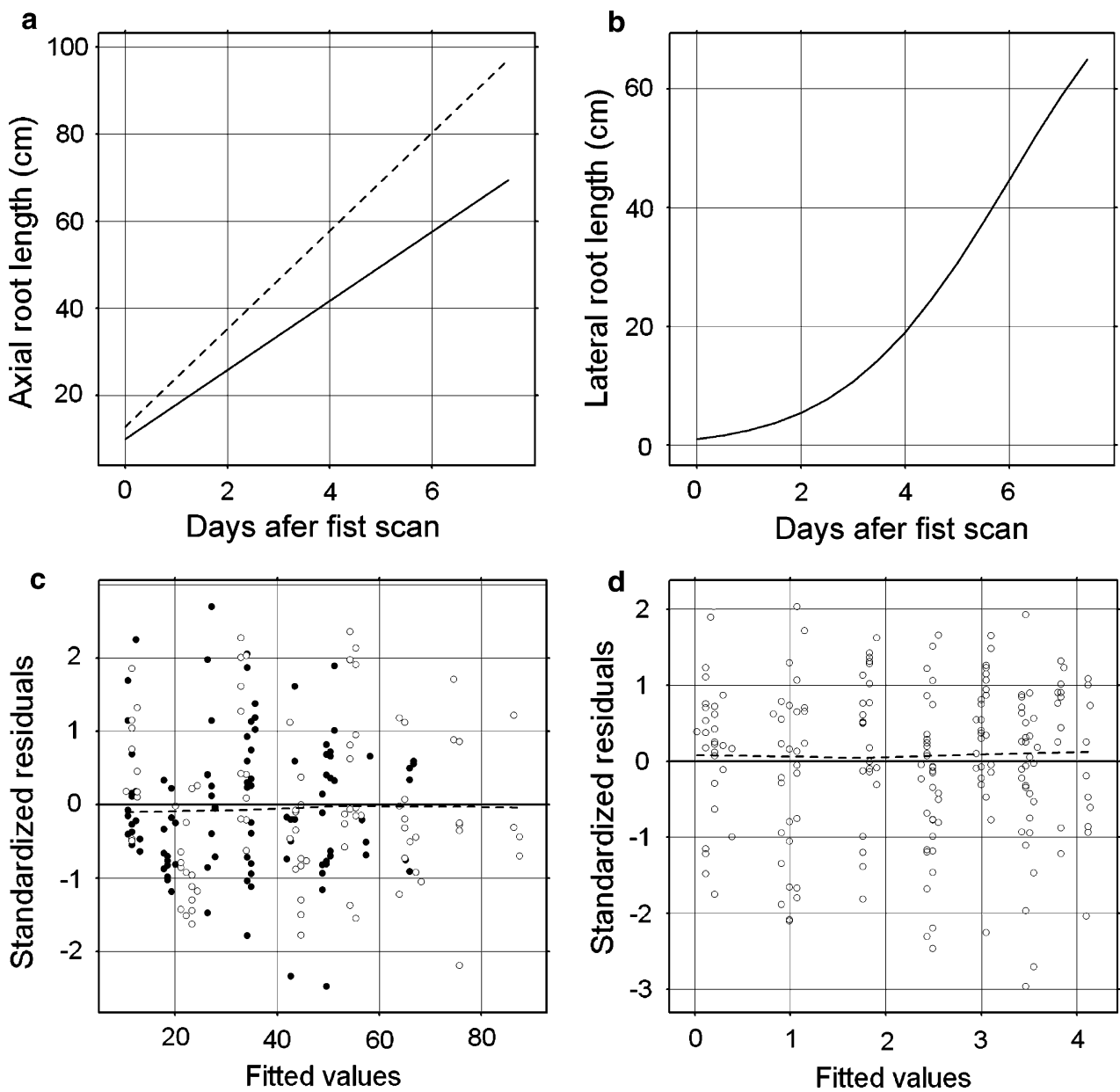

Table 1 Model selection to describe the elongation rates of axile and lateral roots $(P$ values)

\begin{tabular}{|c|c|c|c|c|}
\hline & \multicolumn{2}{|c|}{ Axile roots } & \multicolumn{2}{|c|}{ Lateral roots } \\
\hline & Full & Final & Full & Final \\
\hline Intercept & 0.002 & $<0.001$ & 0.681 & 0.901 \\
\hline Parent line $(P)$ & 0.832 & - & 0.396 & - \\
\hline Water treatment $(W)$ & 0.234 & - & 0.932 & - \\
\hline Time $(T)$ & 0.683 & $<0.001$ & 0.012 & $<0.001$ \\
\hline$T^{2}$ & 0.189 & - & 0.467 & 0.001 \\
\hline$P \times W$ & 0.642 & - & 0.966 & - \\
\hline$P \times T$ & 0.570 & 0.012 & 0.547 & - \\
\hline$W \times T$ & 0.255 & - & 0.862 & - \\
\hline$P \times T^{2}$ & 0.793 & - & 0.827 & - \\
\hline$W \times T^{2}$ & 0.180 & - & 0.987 & - \\
\hline$P \times W \times T$ & 0.775 & - & 0.592 & - \\
\hline$P \times W \times T^{2}$ & 0.716 & - & 0.752 & - \\
\hline
\end{tabular}

The full model (Eq. 1) was reduced stepwise to obtain the final model. This in turn was used to select the most appropriate growth model (Eqs. 2 or 3) watered plants. As $L_{\mathrm{Lat}}$ increased relatively more than $L_{\mathrm{Ax}}$ in WS, $L_{\mathrm{Lat}} / L_{\mathrm{Ax}}$ increased by $49 \%$; since $\mathrm{ER}_{\mathrm{Ax}}$ decreased relatively more than $k_{\mathrm{Lat}}$ in $\mathrm{WS}, k_{\mathrm{Lat}} / \mathrm{ER}_{\mathrm{Ax}}$ showed a relative increase of $20 \%$.

The parents differed in root morphology: $\mathrm{P} 2$ showed a $38 \%$ increase in axile root growth compared to P1; despite this, $\mathrm{P} 2$ had similar numbers of seminal and crown roots at the end of the experiment (Ruta et al. 2009). The stronger growth of axile roots did not result in a significant increase in $k_{\mathrm{Lat}}$ and, as a result. $k_{\mathrm{Lat}} / \mathrm{ER}_{\mathrm{Ax}}$ of $\mathrm{P} 2$ was reduced by onethird compared to that of $\mathrm{P} 1$.

Significant differences in all traits were detected among genotypes. However, significant genotype-by-water treatment interactions were found only for $\mathrm{ER}_{\mathrm{Ax}}$ (Table 2). Heritability ranged from 0.58 for $k_{\mathrm{Lat}}$ to 0.75 for $\mathrm{ER}_{\mathrm{Ax}}$ and $L_{\mathrm{Ax}}$; it was slightly lower under WS, ranging from 0.57 for $k_{\text {Lat }}$ to 0.67 for $k_{\mathrm{Lat}} / \mathrm{ER}_{\mathrm{Ax}}$ and $L_{\mathrm{Ax}}$ (Table 2).

We correlated growth rates with the number of seminal and crown axile roots (data not shown) to elucidate their interdependence. The $\mathrm{ER}_{\mathrm{Ax}}$ correlated with the number of 
Table 2 Summarized statistics of the average values for the following traits of the parental lines and the RILs: rate constant for lateral root elongation $\left(k_{\mathrm{Lat}}\right)$, elongation rate of axile root $\left(\mathrm{ER}_{\mathrm{Ax}}\right)$ and their ratio $\left(k_{\mathrm{Lat}} / \mathrm{ER}_{\mathrm{Ax}}\right)$; lateral root length at the $\mathrm{V} 1$ stage $\left(L_{\mathrm{Lat}}\right)$, axile root length at the V1 stage $\left(L_{\mathrm{Ax}}\right)$ and their ratio $\left(L_{\mathrm{Lat}} / L_{\mathrm{Ax}}\right)$

\begin{tabular}{|c|c|c|c|c|c|c|c|c|c|c|}
\hline \multirow[t]{2}{*}{ Trait } & \multicolumn{3}{|c|}{ Parental lines } & \multicolumn{4}{|l|}{ RILs } & \multicolumn{3}{|c|}{$P$ value $^{\mathrm{a}}$} \\
\hline & P1 & $\mathrm{P} 2$ & & Mean & Min & $\operatorname{Max}$ & $H^{2 \mathrm{~b}}$ & G & $\mathrm{E}$ & $\overline{G \times E}$ \\
\hline 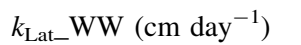 & 0.67 & 0.58 & $\mathrm{NS}^{\mathrm{c}}$ & 0.59 & 0.46 & 0.75 & 0.58 & $* * *$ & $* * *$ & NS \\
\hline$k_{\text {Lat_WS }}\left(\mathrm{cm} \mathrm{day}^{-1}\right)$ & 0.52 & 0.49 & NS & 0.46 & 0.38 & 0.58 & 0.57 & & & \\
\hline $\mathrm{ER}_{\mathrm{Ax}-} \mathrm{WW}\left(\mathrm{cm} \mathrm{day}^{-1}\right)$ & 11.16 & 15.37 & $*$ & 14.56 & 9.19 & 24.78 & 0.75 & $* * *$ & $* * *$ & $* * *$ \\
\hline $\mathrm{ER}_{\mathrm{Ax}-} \mathrm{WS}\left(\mathrm{cm} \mathrm{day}^{-1}\right)$ & 7.87 & 10.93 & $* *$ & 9.53 & 7.30 & 12.96 & 0.60 & & & \\
\hline$k_{\mathrm{Lat}} / \mathrm{ER}_{\mathrm{Ax} \_} \mathrm{WW}$ & 0.0586 & 0.0369 & $*$ & 0.0411 & 0.0213 & 0.0707 & 0.66 & $* * *$ & $* * *$ & NS \\
\hline$k_{\mathrm{Lat}} / \mathrm{ER}_{\mathrm{Ax} \mathrm{x}_{-}} \mathrm{WS}$ & 0.0679 & 0.0450 & $* *$ & 0.0493 & 0.0315 & 0.0742 & 0.67 & & & \\
\hline$L_{\text {Lat_WW }(\mathrm{cm})}$ & 31.68 & 32.35 & NS & 35.03 & 18.09 & 76.81 & 0.72 & $* * *$ & $* * *$ & NS \\
\hline$L_{\text {Lat_WS }(\mathrm{cm})}$ & 47.18 & 66.67 & $*$ & 51.60 & 33.08 & 75.48 & 0.60 & & & \\
\hline$L_{\mathrm{Ax}-} \mathrm{WW}(\mathrm{cm})$ & 51.69 & 65.62 & $* *$ & 63.45 & 40.31 & 110.92 & 0.75 & $* * *$ & NS & NS \\
\hline$L_{\mathrm{Ax}-} \mathrm{WS}(\mathrm{cm})$ & 53.18 & 73.09 & $* *$ & 65.01 & 46.33 & 88.26 & 0.67 & & & \\
\hline$L_{\mathrm{Lat}} / L_{\mathrm{Ax}-\mathrm{WW}}$ & 0.54 & 0.45 & NS & 0.52 & 0.33 & 0.86 & 0.44 & $* * *$ & $* * *$ & NS \\
\hline$L_{\mathrm{Lat}} / L_{\mathrm{Ax}-} \mathrm{WS}$ & 0.83 & 0.92 & NS & 0.77 & 0.48 & 1.16 & 0.38 & & & \\
\hline 30 grains weight $(\mathrm{g})$ & & & & 9.78 & 5.54 & 14.42 & & & & \\
\hline
\end{tabular}

The experiments were performed under well-watered (WW) and water-stressed (WS) conditions

a Statistically difference for the effect of the RILs $(\mathrm{G})$, the water treatment $(\mathrm{E})$ and their interaction $(\mathrm{G} \times \mathrm{E})$

b Broad-sense heritability according to Eq. 4

c Statistical difference between parental lines

$* P$ values $<0.05, * * 0.01, * * * 0.001, N S$ not significant

seminal ( $r=0.44$ for WW and 0.55 for WS) and crown $\left(r=0.28\right.$ for WW and WS) roots. By contrast, $k_{\mathrm{Lat}} / \mathrm{Er}_{\mathrm{Ax}}$ was negatively correlated with the number of seminal roots ( $r=-0.38$ for WW and -0.47 for WS). This indicates that the seminal roots contributed less to the development of the lateral roots compared to the contribution of the primary roots.

\section{Detected QTLs}

To assess the stability of QTLs across treatments, phenotypic data obtained under WW and WS were analyzed jointly for each trait (Table 3). The QTLs were separated into those affecting overall root length, i.e. both axile and lateral root development, and those affecting either root type. Furthermore, a significant QEI indicated whether the trait locus responded to the WS treatment.

QTLs for the modeled elongation rates and for the lengths at the V1 stage co-located for axile roots only

Two of the three QTLs identified for $\mathrm{ER}_{\mathrm{Ax}}$ were the same as QTLs for $L_{\mathrm{Ax}}$ in bins 2.02 and 3.05, with the same algebraic signs of additive effects for both traits (Table 3 ). Two QTLs for $\mathrm{ER}_{\mathrm{Ax}}$ in bins 2.02 and 5.02 were significant for QEI (LOD $<0.8$ ), whereas no corresponding significance was detected for $L_{\mathrm{Ax}}$. There were no common QTLs among $k_{\mathrm{Lat}}$ and $L_{\mathrm{Lat}}$. Between $k_{\mathrm{Lat}} / \mathrm{ER}_{\mathrm{Ax}}$ and $L_{\mathrm{Lat}} / L_{\mathrm{Ax}}$ one co-location was detected in bins 6.04-6.05, even though the traits were only moderately correlated ( $r=0.44$ for WW and 0.56 for WS).

Two major loci, one for vigor (bin 2.02) and one for the relative change in lateral roots (bins 6.04-6.05)

Two major loci were detected, here defined as harboring many traits or traits with a proportion of explained variation around $10 \%$. One QTL affected overall plant growth (bin 2.02), the other the ratio between axile and lateral roots (bins 6.04-6.05). The locus in bin 2.02 affected $E_{\mathrm{Ax}}$ and $L_{\mathrm{Ax}}$ as well as $L_{\mathrm{Lat}}$; the favorable allele was always from P1. At the same location, QTLs for root and shoot dry weight as well as for leaf area were also mapped (Ruta et al. 2009). Therefore, the locus is referred to as a vigor locus. At this locus, the QEI was significant for $\mathrm{ER}_{\mathrm{Ax}}$. In general, the LOD score and the explained variance at this locus were higher under WS, indicating that the increase in vigor was accompanied by an increase in tolerance to low water potential.

The other major locus (bins 6.04-6.05) affected $k_{\mathrm{Lat}}$, $L_{\mathrm{Lat}} / L_{\mathrm{Ax}}$, and $k_{\mathrm{Lat}} / \mathrm{ER}_{\mathrm{Ax}}$ for both WW and WS. The traitincreasing alleles were all from P2. These QTLs yielded the highest LOD scores (4.68-6.5) and PVE values (7.4-14.3). Since the closest marker (gsr1 mapped on chromosome 6) 
Table 3 QTLs detected (Joint LOD > 3.0) for the rate constant of lateral root elongation $\left(k_{\mathrm{Lat}}\right)$, elongation rate of axile roots $\left(\mathrm{ER}_{\mathrm{Ax}}\right)$, and their ratio $\left(k_{\mathrm{Lat}} / \mathrm{ER}_{\mathrm{Ax}}\right)$; lateral root length at the V1 stage $\left(L_{\mathrm{Lat}}\right)$, axile root length at the V1 stage $\left(L_{\mathrm{Ax}}\right)$ and their ratio $\left(L_{\mathrm{Lat}} / L_{\mathrm{Ax}}\right)$

\begin{tabular}{|c|c|c|c|c|c|c|c|c|c|c|c|}
\hline \multirow[t]{2}{*}{ Trait } & \multirow[t]{2}{*}{$\mathrm{Chr} / \mathrm{Bin}$} & \multirow[t]{2}{*}{$\mathrm{cM}$} & \multirow[t]{2}{*}{ Marker } & \multicolumn{4}{|c|}{ LOD score } & \multirow[t]{2}{*}{ Interval } & \multirow[t]{2}{*}{ A } & \multicolumn{2}{|l|}{ PVE } \\
\hline & & & & Joint & WW & WS & QEI & & & WW & WS \\
\hline$k_{\text {Lat }}$ & $6.04-6.05$ & 96.31 & gsr1 & 6.14 & 3.62 & 5.11 & 0.22 & $74-119$ & -0.02 & 9 & 11.8 \\
\hline \multirow[t]{4}{*}{$\mathrm{ER}_{\mathrm{Ax}}$} & 2.02 & 28.51 & umc53a & 3.43 & 2.28 & 2.91 & $1.11 *$ & $4-50$ & 0.30 & 6.8 & 8.1 \\
\hline & 3.05 & 103.21 & csu134d(thf) & 3.56 & 0.38 & 3.36 & 0.01 & $78-134$ & -0.33 & 4.2 & 5.9 \\
\hline & 5.02 & 46.91 & umc $107 \mathrm{~b}$ (croc) & 3.44 & 2.41 & 2.86 & $1.23^{*}$ & $26-63$ & 0.26 & 4.9 & 5.8 \\
\hline & & & & & & & & & & 11.6 & 19.9 \\
\hline$k_{\mathrm{Lat}} / \mathrm{ER}_{\mathrm{Ax}}^{\mathrm{a}}$ & $6.04-6.05$ & 92.3 & gsr1 & 6.2 & 3.8 & 5.3 & 0.0 & $65-109$ & -0.078 & 10.8 & 13.9 \\
\hline \multirow[t]{3}{*}{$L_{\text {Lat }}$} & 2.02 & 16.51 & umc53a & 3.9 & 2.9 & 3.2 & 0.01 & 4-39 & 2.29 & 3.5 & 4.5 \\
\hline & 3.06 & 137.31 & bn18.01 & 3.5 & 3.1 & 2.5 & 0.10 & $119-152$ & -2.39 & 6.8 & 5.8 \\
\hline & & & & & & & & & & 10 & 10 \\
\hline \multirow[t]{4}{*}{$L_{\mathrm{Ax}}$} & 2.02 & 26.51 & umc53a & 3.12 & 1.75 & 3.01 & 0.13 & $4-45$ & 2.53 & 5.6 & 8.2 \\
\hline & 3.05 & 107.11 & $\operatorname{csu} 134 d($ thf $)$ & 3.10 & 0.17 & 2.40 & 0.69 & $80-114$ & -1.89 & 0.4 & 5.4 \\
\hline & 7.05 & 144.71 & umc91a & 3.05 & 2.00 & 2.94 & 0.14 & $128-149$ & -2.51 & 5.1 & 6.9 \\
\hline & & & & & & & & & & 10.3 & 19.1 \\
\hline \multirow[t]{4}{*}{$L_{\mathrm{Lat}} / L_{\mathrm{Ax}}^{\mathrm{b}}$} & 2.06 & 141.1 & umc98a & 4.1 & 4.0 & 0.9 & $0.9^{*}$ & $127-157$ & 0.019 & 9.8 & 2.3 \\
\hline & $6.04-6.05$ & 98.3 & gsr1 & 6.5 & 4.7 & 5.2 & 0.2 & 69-121 & -0.029 & 13.0 & 14.3 \\
\hline & 7.04 & 115.5 & bn18.39 & 3.5 & 0.0 & 2.4 & $2.9^{*}$ & $100-136$ & 0.008 & 0.0 & 6.2 \\
\hline & & & & & & & & & & 21.9 & 21.6 \\
\hline
\end{tabular}

* Significant QTL-by-water treatment interaction at LOD $>0.8$

The QTL characteristics include the chromosome and bin number (Chr/Bin); the position of the QTL peak in cM; the LOD score for the joint analysis, the individual water treatment and the QTL-by-water treatment interaction (QEI); the confidence interval in cM in which the LOD score dropped by half; the additive contribution of the P1 allele (A); the percentage of phenotypic variance explained (PVE; $\left.R^{2}\right)$ by the individual QTLs within each water treatment and considering all the significant QTLs (total PVE)

${ }^{a}$ Log-transformed

b Square root-transformed

has not yet been introduced into the Maize Genetics and Genomics Database (Lawrence et al. 2008), we present here the locus in bin 6.04 and 6.05 (6.04-6.05), where two flanking makers umc113b and csu60a, respectively, were located.

Loci controlling lateral roots were not affected by low water potential

In general, $k_{\mathrm{Lat}}$ and $L_{\mathrm{Lat}}$ were not responsive to water stress. A locus for $L_{\text {Lat }}$ was detected in bin 3.06 that was linked to a constitutive locus for $\mathrm{ER}_{\mathrm{Ax}}$ and $L_{\mathrm{Ax}}$ in bin 3.05. All traitincreasing alleles were contributed by $\mathrm{P} 2$. Another locus in bin 2.06 did not show a significant response to WS but changed the $L_{\mathrm{Lat}} / L_{\mathrm{Ax}}$.

Loci controlling $\mathrm{ER}_{\mathrm{Ax}}$ and $L_{\mathrm{Lat}} / L_{\mathrm{Ax}}$ were affected by low water potential

As expected from the genotype-by-water treatment interaction, $\mathrm{ER}_{\mathrm{Ax}}$ was one of the traits that showed a significant QEI at two of three loci (bins 2.02 and 5.02). Among the three loci detected for $L_{\mathrm{Lat}} / L_{\mathrm{Ax}}$, two responded to water stress. The locus in bin 2.06 was specific to WW conditions, while the locus in bin 7.04 was specific to WS. In both cases, P1 contributed the trait-increasing allele.

QTL co-locations: comparison with other studies of the same population

The QTLs for elongation rates of roots were compared with elongation rates for leaves and silks published by Welcker et al. (2007) and the ASI published by Ribaut et al. (1996). The ASI is used as a proxy measure of silk elongation rates (Welcker et al. 2007). At the major QTL in bins 6.04-6.05, the increase in $k_{\text {Lat }}$ and the ratio between lateral and axile roots was co-located with an increase in ASI (Fig. 2). Furthermore, a WW-specific QTL for $L_{\mathrm{Lat}} / L_{\mathrm{Ax}}$ overlapped with QTLs for ASI under WW and WS in bins 2.06-2.07. The directions of the co-locating QTLs, however, were opposite (Fig. 2). There was no close co-location between QTLs for the root length traits in this study and the leaf elongation rates (LER) detected by Welcker et al. (2007). However, a QTL for $\mathrm{ER}_{\mathrm{Ax}}$ in bin 5.02 was $30 \mathrm{cM}$ from the QTLs for LER in response to evaporative demand (LERb) 


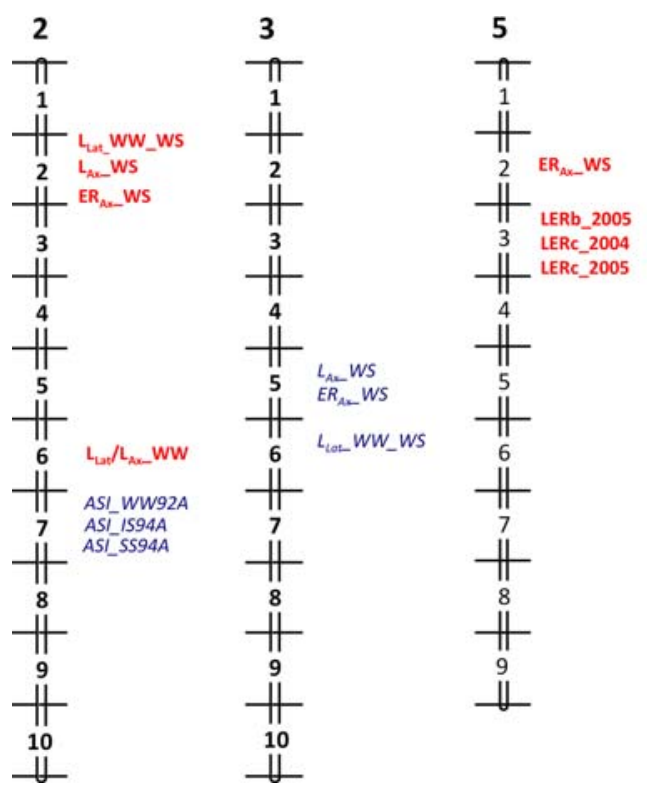

Fig. 2 Co-locations of QTLs for root growth in seedling stage in this study (see Table 3 for explanation of abbreviations) with QTLs for anthesis-silking interval (ASI) identified by Ribaut et al.(1996) and QTLs for leaf elongation rate (LER) identified by Welcker et al. (2007) All QTLs were identified in P1 $\times$ P2 population. Letters in

and soil water deficit (LERc). The P1 allele contributed to the increase in $\mathrm{ER}_{\mathrm{Ax}}, \mathrm{LERb}$, and LERc under water stress.

\section{Discussion}

The applied moderate water stress of $-0.74 \mathrm{MPa}$ (measured as leaf water potential), induced by $20 \%$ PEG, was well above the permanent wilting point of $-1.5 \mathrm{MPa}$. As the leaf water potential was measured under non-transpiring conditions, it reflects the water potential in the pouches. The severity of the stress was set to hamper the elongation of axile and lateral roots. In preliminary studies, we observed enhanced growth of lateral roots at a lower concentration of $15 \%(\mathrm{w} / \mathrm{v})$ and increased the stress level accordingly. The parental inbred lines differed similarly with regard to early and late root morphology. The inbred line P2 showed enhanced growth of axile roots and a lower ratio between the lateral and axile roots, in line with observations in growth-column experiments (Hund et al. 2009a). In growth columns at the V5 stage, P2 had a lower lateral-to-axile root ratio and a greater specific proportion of deep roots compared to $\mathrm{P} 1$.

Which are the interesting loci for altering the root morphology in the studied population?

The vigor locus in bin 2.02, showing a response to water stress, can be utilized for further genetic analyses with the

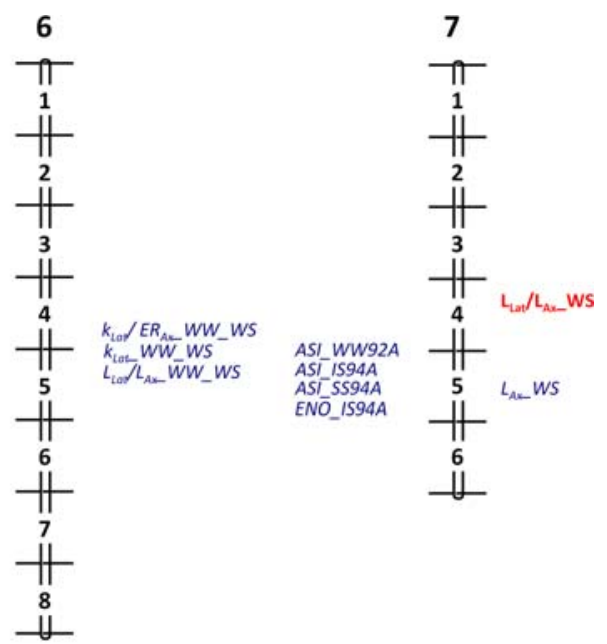

bold and italic indicate an increasing allele contributed by P1 and P2, respectively. Environments are indicated as follows: well-watered (WW), water-stressed (WS), both well-watered and water-stressed (WW_WS), intermediate stressed (IS) and severe stressed (SS). For Field traits, only matching QTL are presented

aim of improving general plant growth, even under early unfavorable conditions. Early vigor of hybrids released in the US Corn Belt from 1930 to 2000 (era hybrids of Pioneer Hi-Bred International) showed a linear decrease in root and shoot weight (Sanguineti et al. 2006). Obviously, breeders selected against early vigor, which may, in part, be a consequence of the adaptation of maize to higher plant densities. Nevertheless, vigor loci, such as the one detected here, can be utilized in environments with early drought, since they allow for a rapid plant establishment and canopy closure, as discussed by Richards et al. (2002).

The ratio between axile and lateral roots changed due to low water potential. As outlined in the introduction, rooting depth can be increased by redirecting the allocation of resources from lateral to axile roots. The locus in bin 6.046.05 is interesting, because a decrease in $k_{\mathrm{Lat}} / \mathrm{ER}_{\mathrm{Ax}}$ was associated with a decrease in $k_{\text {Lat }}$ and an increase in the number of seminal roots (Ruta et al. 2009) as well as with drought tolerance in the field as indicated by a lower ASI (Ribaut et al. 1996). These differences in the organization of the embryonic root system (primary lateral vs. seminal axile roots) are typical for maize (Hund et al. 2007, 2009b). The importance of seminal roots is supported by the fact that the yield of the era hybrids of Pioneer was negatively correlated with vigor (shoot and root weight) and the weight of the primary root but not with the weight of the seminal roots (Sanguineti et al. 2006). Furthermore, of all root the QTLs observed by Tuberosa et al. (2002), those for 
the weight of seminal roots showed the most consistent association with grain yield. In wheat, too, a greater number of seminal roots can make a significant contribution to water uptake (Manschadi et al. 2008).

Is early root morphology related to elongation rates of leaves and silks?

The growth of silks and leaves in the $\mathrm{P} 1 \times \mathrm{P} 2$ population has common genetic determinisms, as suggested by the results of Welcker et al. (2007). Half the QTLs for these two traits were common in both well-watered and water-deficit conditions. We assumed a common genetic determinism for leaves, silks and roots. While this was the case for roots and silks (ASI in bin 6.04-6.05; Ribaut et al. 1996), it was not the case for roots and leaves. This lack of co-location under well-watered conditions might be because root traits were measured during the heterotrophic stage, while the leaves were measured during the autotrophic stage. Common QTLs for root and leaf growth is expected at later stages when all carbohydrates are derived from leaves.

The lack of QTL co-locations for roots and leaves in response to low water potential may be due to the timing of the stress. The rapid changes in water potential due to the application of PEG led to rapid dehydration invoking fast response pathways (Verslues et al. 2006). However, about $24 \mathrm{~h}$ after this "acute" phase, the typical long-term responses to low water potential can be observed. These include solute accumulation and osmotic adjustment (Verslues and Bray 2004) and similar changes in root and shoot growth (van der Weele et al. 2000) as occurs in soil. Therefore, a more likely explanation for the lack of QTL co-locations is that the control mechanisms differ. For example, roots still elongate at water potentials lower than $-1.5 \mathrm{MPa}$ (Sharp et al. 1988), at which leaf elongation in the P1 × P2 population ceases (Welcker et al. 2007). This suggests a different genetic control of the responses of roots and shoots to low water potential, which are optimized to enhance avoidance, i.e. prolonged elongation of the roots (water source) and a more rapid decrease in the elongation of the leaves (water sinks).

Concerning co-locations for roots and silks, the major locus in bins 6.04-6.05 was responsible for the constitutive increase in the ASI (Ribaut et al. 1996) as well as for the constitutive increase in the length of lateral roots. We assume that $k_{\text {Lat }}$ is dominated by the primary lateral roots (see Hund et al. 2009b). The weight of the primary root is, in turn, negatively associated with the historic yield increases in the era hybrids of Pioneer (Sanguineti et al. 2006) as outlined above. The negative co-location of $k_{\text {Lat }}$ and drought tolerance (narrow ASI) is therefore not surprising.
What are the advantages of measuring growth rates?

It is time-consuming to assess growth rates, which involves scanning the root system at regular intervals, but do these assessments have advantages over simpler evaluations? Growth dynamics provide information about the response of roots to applied stresses and enable correction for differences in germination. Errors in root length due to differences in germination can be large, particularly for the lateral roots, which grow exponentially (Hund et al. 2009b). It is difficult to control these errors in QTL populations, since differences in germination are usually unknown before evaluation and cannot be integrated into the experimental design. Despite the fact that these differences in germination in the present population were small (not more than $6 \mathrm{~h}$ ), they might have influenced $L_{\text {Lat }}$. Thus, while we are sure that the QTL for $k_{\text {Lat }}$ (bin 6.04-6.05) is not due to differences in germination, we are not certain that this is the case for the QTLs for $L_{\text {Lat }}$.

Assessing the dynamics led to a more precise determination of the response to stresses. For example, $E_{\mathrm{Ax}}$ responded significantly to water stress, while $L_{\mathrm{Ax}}$ did not. Here, the dynamic accounted for plant-to-plant variability at the start of the stress treatment. Thus, in this type of study, an assessment of the dynamic traits yielded more reliable results than an assessment of cumulated traits.

\section{Conclusion}

Growth pouches, used in this study, enabled the measurement of the elongation rates of roots and enabled us to detect interactions between genotypes/QTLs and the water treatment. The positive effect of the P1 allele on the growth of axile roots under WS (vigor locus in bin 2.02) indicates that it may be used to increase tolerance during early periods of drought. The candidate locus in bins 6.04-6.05 can change the embryonic root by decreasing the growth of lateral roots while increasing the amount of seminal axile roots. This may enhance drought avoidance by increasing the number of deep-reaching roots and is evidenced by the co-location to a short ASI and consistent findings in the literature. Further efforts are necessary to elucidate the impact of these loci on root morphology at later stages of development.

Acknowledgments The authors thank Dr. Jean-Marcel Ribaut and Prof. Dr. François Tardieu for the helpful discussions, Marcia Schoenberg for linguistic corrections, Dr. Susanne Stamp for handling the manuscript, and the Generation Challenge Program (GCP) for stimulating interactions and financial support. 


\section{References}

Barreto HJ, Edmeades GO, Chapman SC, Crossa J (1997) The alpha lattice design in plant breeding and agronomy. Generation and analysis. In: Edmeades GO et al (eds) Developing drought and low N-tolerant maize. Proceedings of a Symposium, El Batan, Mexico. 25-29 March 1996. CIMMYT, Mexico DF, pp 544-551

Basten CJ, Weir BS, Zeng Z (2003) QTL Cartographer. Version 1.17. A reference manual and tutorial for QTL mapping. Department of Statistics. North Carolina State University, Raleigh

Bolaños J, Edmeades GO, Martinez L (1993) Eight cycles of selection for drought tolerance in lowland tropical maize. III. Responses in drought-adaptive physiological and morphologial traits. Field Crops Res 31:269-286

Bruce WB, Edmeades GO, Baker TC (2002) Molecular and physiological approaches to maize improvement for drought tolerance. J Exp Bot 53:13-25

Butler DG, Cullis BR, Gilmour AR, Gogel BJ (2007) Analysis of mixed models for $\mathrm{S}$ language environments. ASReml-R reference manual, Technical report, Queensland Department of Primary Industries

Campos H, Cooper A, Habben JE, Edmeades GO, Schussler JR (2004) Improving drought tolerance in maize: a view from industry. Field Crops Res 90:19-34

Carpita N, Sabularse D, Montezinos D, Delmer DP (1979) Determination of the pore size of call walls of living plant cells. Science 20:1144-1147

Fracheboud Y, Ribaut J-M, Vargas M, Messmer R, Stamp P (2002) Identification of quantitative trait loci for cold-tolerance of photosynthesis in maize (Zea mays L.). J Exp Bot 53:1967-1977

Fuad-Hassan A, Tardieu F, Turc O (2008) Drought-induced changes in anthesis-silking interval are related to silk expansion: a spatiotemporal growth analysis in maize plants subjected to soil water deficit. Plant Cell Environ 31:1349-1360

Giuliani S, Sanguineti MC, Tuberosa R, Bellotti M, Salvi S, Landi P (2005) Root-ABA1, a major constitutive QTL, affects maize root architecture and leaf ABA concentration at different water regimes. J Exp Bot 56:3061-3070

Hallauer AR, Miranda JB (1981) Quantitative genetics in maize breeding, 1st edn. Iowa State University Press, Ames

Hammer GL, Dong Z, McLean G, Doherty A, Messina C, Schussler J, Zinselmeier C, Paszkiewicz S, Cooper M (2009) Can changes in canopy and/or root cystem architecture explain historical maize yield trends in the U.S. Corn Belt? Crop Sci 49:299-312

Hund A, Frachboud Y, Soldati A, Frascaroli E, Salvi S, Stamp P (2004) QTL controlling root and shoot traits of maize seedlings under cold stress. Theor Appl Genet 109:618-629

Hund A, Richner W, Soldati A, Fracheboud Y, Stamp P (2007) Root morphology and photosynthetic performance of maize inbred lines at low temperature. Eur J Agron 27:52-61

Hund A, Ruta N, Liedgens M (2009a) Rooting depth and water use efficiency of tropical maize inbred lines, differing in drought tolerance. Plant Soil 318:311-325

Hund A, Trachsel S, Stamp P (2009b) Growth of axile and lateral roots of maize: I development of a phenotyping platform. Plant Soil. doi:10.1007/s11104-009-9984-2

Jiang CA, Zeng ZB (1995) Multiple-trait analysis of genetic-mapping for quantitative trait loci. Genetics 140:1111-1127

Kirkegaard JA, Lilley JM, Howe GN, Graham JM (2007) Impact of subsoil water use on wheat yield. Aust J Agric Res 58:303-315

Lagerwer J, Eagle HE, Ogata G (1961) Control of osmotic pressure of culture solutions with polyethylene glycol. Science 133:1486

Lawrence C, Harper L, Schaeffer M, Sen T, Seigfried T, Campbell D (2008) The Maize Model Organism Database for basic, translational, and applied research. Int $\mathrm{J}$ Plant Genomics. 2008:496957

Liu JC, Li JS, Chen FJ, Zhang FS, Ren TH, Zhuang ZJ, Mi GH (2008) Mapping QTLs for root traits under different nitrate levels at the seedling stage in maize (Zea mays L.). Plant Soil 305:253-265

Manschadi AM, Christopher J, deVoil P, Hammer GL (2006) The role of root architectural traits in adaptation of wheat to waterlimited environments. Funct Plant Biol 33:823-837

Manschadi AM, Hammer GL, Christopher JT, deVoil P (2008) Genotypic variation in seedling root architectural traits and implications for drought adaptation in wheat (Triticum aestivum L.). Plant Soil 303:115-129

Pinheiro J, Bates D, DebRoy S, Sarkar D (2007) The R Development Core Team nlme, linear and nonlinear mixed effects models. $\mathrm{R}$ package version 3:1-85. http://CRAN.R-project.org/

Ribaut JM, Hoisington D, Deutsch JA, Jiang C, Gonzales de Leon D (1996) Identification of quantitative trait loci under drought conditions in tropical maize. 1. Flowering parameters and the anthesis-silking interval. Theor Appl Genet 92:905-914

Ribaut JM, Jiang C, Gonzalez-de-Leon D, Edmeades GO, Hoisington DA (1997) Identification of quantitative trait loci under drought conditions in tropical maize.II. Yield components and markerassisted selection strategies. Theor Appl Genet 92:887-896

Ribaut JM, Fracheboud Y, Monneveux P, Bänziger M, Vargas M, Jiang CJ (2007) Quantitative trait loci for yield and correlated traits under high and low soil nitrogen conditions in tropical maize. Mol Breed 20:15-29

Ribaut JM, Betrán FJ, Monneveux P, Setter T (2008) Drought tolerance in maize. In: Hake SC, Bennetzen JL (eds) Hand book of maize: its biology. Springer, Netherlands, pp 311-344

Richards RA, Rebetzke GJ, Condon AG, van Herwaarden AF (2002) Breeding opportunities for increasing the efficiency of water use and crop yield in temperate cereals. Crop Sci 42:111-121

Ruta N, Stamp P, Liedgens M, Fracheboud Y, Hund A (2009) Collocation of QTLs for seedling traits and yield components of tropical maize under limited water availability. Crop Sci (in press)

Sanguineti MC, Duvick DN, Smith S, Landi P, Tuberosa R (2006) Effects of long-term selection on seedling traits and ABA accumulation in commercial maize hybrids. Maydica 51:329-338

Sharp RE, Davies WJ (1985) Root growth and water uptake by maize plants in drying soil. J Exp Bot 36:1441-1456

Sharp RE, Davies WJ (1989) Regulation of growth and development of plants growing with a restricted supply of water. In: Jones HG, Flowers TL, Jones MB (eds) Plants under stress. Cambridge University Press, Cambridge, pp 71-93

Sharp RE, Silk WK, Hsiao TC (1988) Growth of the maize primary root at low water potentials. 1. Spatial-distribution of expansive growth. Plant Physiol 87:50-57

Trachsel S, Messmer R, Stamp P, Hund A (2009) Mapping of QTLs for lateral and axile root growth of tropical maize. Theor Appl Genet. doi:10.1007/s00122-009-1144-9

Tardieu F, Bruckler L, Lafolie F (1992) Root clumping may affect the root water potential and the resistance to soil-root water transport. Plant Soil 140:291-301

Tuberosa R, Sanguineti MC, Landi P, Giuliani MM, Salvi S, Conti S (2002) Identification of QTLs for root characteristics in maize grown in hydroponics and analysis of their overlap with QTLs for grain yield in the field at two water regimes. Plant Mol Biol 48:697-712

van der Weele CM, Spollen WG, Sharp RE, Baskin TI (2000) Growth of Arabidopsis thaliana seedlings under water deficit studied by control of water potential in nutrient-agar media. J Exp Bot $51: 1555-1562$

Verslues PE, Bray EA (2004) LWR1 and LWR2 are required for osmoregulation and osmotic adjustment in Arabidopsis. Plant Physiol 136:2831-2842 
Verslues PE, Agarwal M, Katiyar-Agarwal S, Zhu JH, Zhu JK (2006) Methods and concepts in quantifying resistance to drought, salt and freezing, abiotic stresses that affect plant water status. Plant J 45:523-539

Welcker C, Boussuge B, Bencivenni C, Ribaut JM, Tardieu F (2007) Are source and sink strengths genetically linked in maize plants subjected to water deficit? A QTL study of the responses of leaf growth and of Anthesis-Silking Interval to water deficit. J Exp Bot 58:339-349

Zhu JM, Kaeppler SM, Lynch JP (2005) Mapping of QTLs for lateral root branching and length in maize (Zea mays L.) under differential phosphorus supply. Theor Appl Genet 111:688-695 